\title{
Kreditkarten: \\ Outsourcing steigert die Effizienz
}

\author{
Während Banken ihre Strukturen weiter auf Effizienz trimmen, steigen die Ansprüche der \\ Kunden. Kreditkarten sollen künftig auch kontaktloses Bezahlen am Touch Point ermöglichen \\ und der Service des Unternehmens soll rund um die Uhr erreichbar sein.
}

Viele Institute sind dadurch inzwischen gezwungen, ganze Leistungsbündel auszulagern.

\begin{abstract}
$\mathbf{F}$ rüher war es eher eine Glaubensfrage, Leistungen outzusourcen oder Inhouse zu erledigen“, sagt Steffen Mahlmeister, Leiter Zentrales Client Management bei Swiss Post Solutions (SPS). „Davon kann heute keine Rede mehr sein. Banken, die den Spagat zwischen 100 Prozent Kosteneffizienz bei einem Service-Level von nahezu 100 Prozent schaffen wollen, müssen weite Teile ihrer Wertschöpfungskette outsourcen.“
\end{abstract}

\section{WERTSCHÖPFUNGSKETTE}

Dieser Prozess beginnt bereits bei der Kundengewinnung. Beispiel Kreditkarte: In diesem nahezu gesättigten Markt reichen kreative Ideen alleine nicht mehr aus, um neue Kunden zu gewinnen. Vielmehr müssen Interessenten identifiziert und per Multichannel-Kampagne möglichst individuell angesprochen werden. Dabei unterstützt der auf Business Process Outsourcing (BPO) spezialisierte Dienstleister SPS Banken von Anfang an:

- Neukundengewinnung per MultichannelKampagne

- Konzeption, Aufbau und Implementierung von Datenbanken

- Antragsmanagement inkl. scannen und bearbeiten von bis zu 250 Millionen Dokumenten weltweit pro Jahr

- Eigene Herstellung von Wertkartenrohlinoen in Meitschland

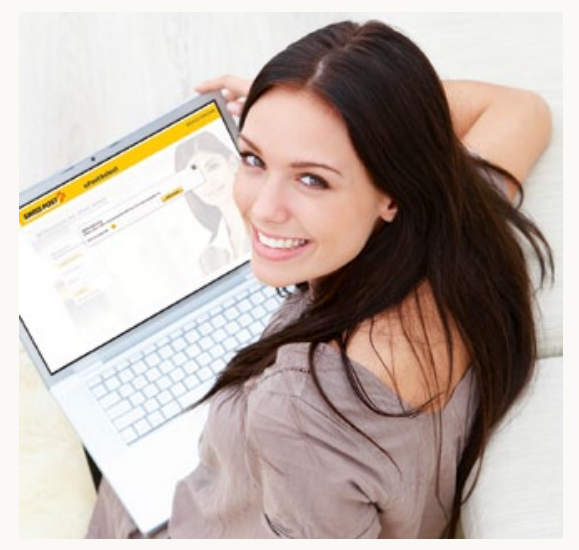

Rechnungsversand physisch oder digital per ePostSelect.

- Neues Chipkartenbetriebssystem JACOS ${ }^{\circledR} 2.0$

- Produktion von bis zu 1,6 Millionen Karten und 250.000 Chip-Karten täglich im größten Kartenpersonalisierungszentrum an einem Standort Europas in Bamberg (Visa- \& MasterCard zertifiziert)

- Customer Contact Management für perfekten Kundenservice

Selbst den monatlichen Druck der Kreditkartenabrechnungen übernimmt SPS im hauseigenen Document Output Center.

\section{ABRECHNUNG AUCH PER E-MAIL}

Neu ist jetzt der Versand der Kreditkartenabrechnung per ePostSelect. Dabei können die Empfänger selbst entscheiden, ob sie ihre Rechnung nach wie vor per Brief oder über die Serure-Mail-I ösuno der Srhweizeri- schen Post ,IncaMail' erhalten möchten. Mi IncaMail können Banken ihre Kreditkartenabrechnungen und andere vertraulich Dokumente sicher per E-Mail versenden. Der Vorteil: Die Umstellung kann sukzessi erfolgen und der Empfänger muss seine bestehende E-Mail-Adresse nicht wechseln

$\pi$ SWISS POST SOLUTIONS

\section{SPS}

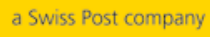

- Swiss Post Solutions (SPS), eine 100-prozentige Tochter der Schweizerischen Post, ist ein führender Anbieter von Business Process Lösungen physisch und digital.

\section{KONTAKT:}

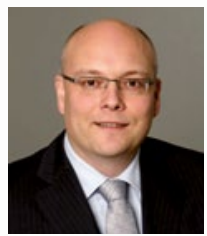

Steffen Mahlmeister Leiter Zentrales Client Management Kronacher Straße 70-80 96052 Bamberg

Tel.: +49 (0) $9519426-3182$ steffen.mahlmeisteraswisspost.com www.swisspostsolutions.de 